\title{
Information Needs at the O.R. Waiting Room
}

\author{
Giuliana COLUSSI ${ }^{\mathrm{a}, 1}$, Eliana FRUTOS ${ }^{\mathrm{a}}$, Romina RAPISARDA ${ }^{\mathrm{a}}$, Janine SOMMER ${ }^{\mathrm{a}}$, \\ Juan DESCALZO ${ }^{a}$, Fernando PLAZZOTTA ${ }^{\mathrm{a}}$ and Daniel LUNA ${ }^{\mathrm{a}}$ \\ ${ }^{a}$ Health Informatics Department, Hospital Italiano de Buenos Aires, Argentina
}

\begin{abstract}
The objective of this study was to investigate the difficulties and information needs that both patients and their companions have in the context of a scheduled surgery. Observations in the operating room waiting zone and semistructured interviews were conducted to patients with scheduled surgeries, their companions, administrative staff members and surgeons. We developed a journey map to explain the patient's experience through the perioperative process. The emerging categories were Fragmented communication, Understanding the surgical process and Waiting times. Knowing the information needs of patients and companions was important to identify problems and opportunities for improvement in our institution's Personal Health Records.
\end{abstract}

Keywords. Personal Health Records, Surgery, Perioperative Experience.

\section{Introduction}

Going through surgery may be a traumatic experience both for the patient and their companions, giving rise to lasting health disorders [1]. The relationship between expectations and the real experience of the surgical process influences the levels of stress and anxiety experienced during the day of the operation. An imbalance in this relationship could negatively impact the patient [2]. Rhodes et al reported in their review that keeping the patient up to date on waiting times, providing information and explaining the different aspects of care decreases stress and anxiety, and increases patient satisfaction [3]. In the same way, Doyle et al highlighted the importance of information and communication as a positive influencing factor. In addition, they reported an association between patient experience and clinical outcomes [4].

While most studies focus on the patient, there is evidence that warns about the anxiety and distress experienced by the patient's family while accompanying them through the surgical process [5]. However, studies focused on the communication of information to these companions in hospitals, especially in the surgical field, are scarce [6].

The implementation of technological tools that provide users with access to their health information has encouraged them to participate more actively in their health management. The arrival of personal health records (PHR) have contributed to this. They ease access to health information and encourage electronic communication between

\footnotetext{
${ }^{1}$ Corresponding Author, Giuliana Colussi, Health Informatics Department, Hospital Italiano de Buenos Aires, Tte. Gral. Juan D. Perón 4190, C1199ABB Buenos Aires, Argentina; E-mail: giuliana.colussi@hospitalitaliano.org.ar.
} 
health professionals and patients in a safe environment [7]. However, the benefits that a PHR gives are often forgotten when the patient is at the hospital. Specially when going through a surgical intervention, where the systems that provide real-time information to the patient and family are limited.

In this line, we set out to investigate the difficulties and/or information needs that both patients and their companions have in the context of a scheduled surgery. The results of this research will contribute to the design of a module within the PHR that provides information to patients and companions before a surgical event.

\section{Methods}

The Hospital Italiano is a high complexity medical center located in the City of Buenos Aires. Each year, it handles 2,800,000 consultations, performs 46,500 discharges and 52,000 surgical procedures in its 41 operating rooms. It has 785 inpatient beds -200 for critical care- and 800 home medicine beds. It employs 9237 people, including 3400 doctors. Since 1998, HIBA has had its own (in-house developed) health information system [8]. An integrated Personal Health Record (PHR) has been available since 2007. Its main functionalities include: appointment scheduling, test results visualization, patient-practitioner secure messaging, health information sharing, management of prescribed medication, and different teleconsultation modalities. At present, it has approximately four hundred thousand registered users [9].

Semi-structured interviews were conducted with patients with scheduled surgeries and their companions in the central operating room (OR) waiting zone, administrative staff members who participated in the patient admission process for these surgeries, and surgeons. All interviews were audio recorded for further analysis, safeguarding the identity of the participants, who participated voluntarily through informed oral consent.

Participants were selected by convenience sampling. The interviews were conducted until theme saturation was achieved. The interviews were transcribed and the analysis material consisted of literal transcripts.

Likewise, observations of the administrative processes were made in the waiting room to describe the phenomenon as it develops in its natural environment, trying to achieve relevant descriptions. During the observation, the research team ethnographically recorded their observations in the most detailed and descriptive way possible, including their own impressions of the studied scenario. This record was included in the material submitted to the analysis process.

The analysis process was carried out simultaneously with data collection, through the coding and categorization of the information, based on a constant comparison process. The categories were defined taking into account the main research questions and the emerging findings from the interviews and observations, building a descriptive framework that helped to contextualize the collected data [10].

The research project was approved by the institutional ethics committee (CEPI \# 5278). The study was performed in full agreement with current national and international ethical regulations. 


\section{Results}

During the months of September and October 2019, the team conducted 11 interviews with patients and companions, 5 with administrative staff and 3 with surgeons. Furthermore, they performed observations in the waiting room of the central operating room at different days and times. A Journey Map (figure 1) was created to understand the complete process from the patient's point of view. Starting from the moment the surgery is decided until they are hospitalized after the operation, identifying the actors and information systems involved at each step, and pointing out in red where the pain points are located.

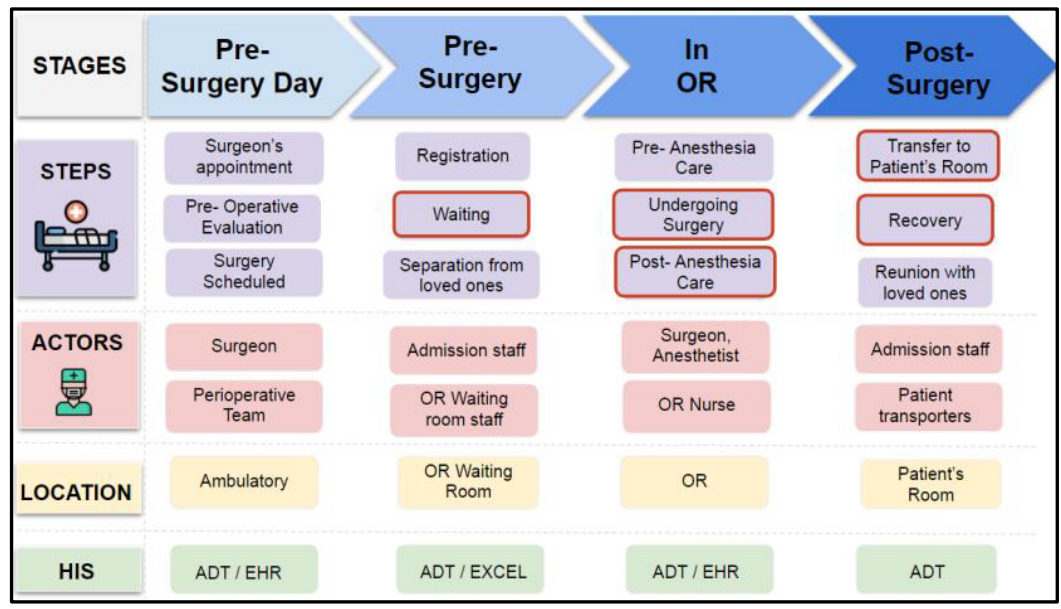

Figure 1. Surgical Process Journey Map

The emerging categories arise from the combination of the techniques used, allowing greater richness in the understanding of the phenomenon. We found three main dimensions relating to the communication between patients and companions, administrative staff and the healthcare team.

The information given to patients in the days before surgery is partial, is communication is diverse and, in some cases, may be contradictory depending on the transmitter. Patients receive instructions from the surgeon and their secretary, the anesthesia team, and the hospital's administrative staff. Each one gives information to the patient in a fragmented and uncoordinated manner. In addition, it is mainly done orally. It is common that, during these stressful situations, people cannot recall exactly what they were told, which causes great concern and anxiety. "I had to take a picture of the piece of paper my surgeon's secretary gave me. In case I lost it." (Patient, 23)

Companions report having information needs about the patient's condition during the time they are waiting for the surgeon's update. While the companions know about the surgical intervention and the duration of the intervention, the entire process within the operating area remains unknown: the time prior to surgery, from the patient leaving the waiting room until the start of the surgery itself; and the time post-surgery, in the recovery room, when the companions receive the surgery update. Both patients and their companions need to know what the entire surgical procedure entails. What happens from the time they arrive at the hospital until they are transferred to a general ward bed post surgery. The surgeons and administrative staff interviewed agreed that this type of information reassures them and lowers anxiety. "My son has been in the operating room 
since 10. It's 2 p.m. now and I haven't had any news (...) the doctor told me that the intervention would last 2 hours" (Patient's mother, 50)

Waiting times were a problem reported by all interviewed groups, especially by patients. Both the length and not knowing the real waiting times were described as problematic. Due to the usual delays, some patients may be waiting for several hours to be called. Usually, fasting for extended periods. Doctors inform patients about the technical issues of the surgery, and about its length. However, they do not give them information about the time that may elapse before entering and after leaving the operating room. The administrative staff informs the patient and companion about a more realistic scenario regarding surgical times. Nevertheless, they already come with biased information, generating misunderstandings and confusion. Waiting times, coupled with fragmented information and a lack of accurate knowledge about the surgical process, increases the stress and anxiety that patients and their companions go through in the context of surgery. "Patients get very nervous when they have to wait longer than they thought" (Administrative staff, 42)

\section{Discussion}

Our research shows that both the patient and their family do not know or do not understand the entire surgical process before the day of surgery. Through the Journey Map we were able to get closer to the users' experience, illustrating the different interactions they have with the health system in relation to the operation. Unlike what is published by Ridder et al [11], our findings show that the surgical experience begins at the time the surgery is prescribed and not the day of the intervention. Various aspects of health care delivery, including communication and information, are closely related to a positive patient experience. The importance of an optimal experience is demonstrated by its relationship with outcomes reported by different studies such as Doyle and colleagues[12]. This is why we designed a new module within our PHR, called "Me tengo que operar" (Spanish for "I have to have surgery"). It will be enabled when a patient has a surgery scheduled, providing clinical and administrative information regarding the operation. Thus, both the patient and his family will arrive at the day of surgery with reliable, complete and unambiguous information about the entire surgical process. In turn, our findings show that the most critical moments in the patient's and family experience were marked by long waiting times and lack of notification about delays. These findings are consistent with research by Pearson et al. [13] and Rhodes et al. [14]. In addition, both show that intervening in these aspects could reduce the negative impact on those affected. These critical moments were also the object of study by various authors for the design of technological tools that provide a solution to this problem. Poudel and colleagues demonstrated that sending SMS messages updating the intrasurgical status reduces the family members anxiety [15]. Similarly, Hodge et al implemented an app for companions that provides constantly updated information. They showed that this improvement in communication has a direct correlation with the highest satisfaction reported by family members [16]. In response to our findings and in accordance with what is reported in the literature, we plan to incorporate within the inpatient's PHR a new module that can be accessed by companions (authorized by the patient), providing synchronous information on the patient's location and status.

While these strategies target patients and companions, it is important not to forget the care staff point of view, to not interfere negatively in their workflow. In this sense, we 
consider that the greatest strength of our work was to integrate the voices of all users who take part in the process, before designing and implementing a new tool.

This study represents a framework from which a feasible and promising user-centric IT solution could be planned. We detected the need for a technological tool that allows patients to access comprehensive and complete information not only of the surgical day, but also about the pre and post phases of the surgical experience that they will go through. We believe that further research should be conducted in this area to assess postimplementation impact: potential benefits, usage and satisfaction.

\section{Conclusion}

Knowing the perceptions of users regarding the information needs of patients and companions in the context of scheduled surgery was valuable as a situational diagnosis to identify problems and opportunities for improvement in our institution's PHR.

\section{References}

[1] Mucci, M.. Psicoprofilaxis quirúrgica: Una práctica en convergencia interdisciplinaria. Paidós (2015)

[2] Costa MJ. The lived perioperative experience of ambulatory surgery patients. AORN J. 2001 Dec;74(6):874-81. doi: 10.1016/s0001-2092(06)61505-6. PMID: 11795060.

[3] Rhodes L, Miles G, Pearson A. Patient subjective experience and satisfaction during the perioperative period in the day surgery setting: a systematic review. Int J Nurs Pract. 2006 Aug;12(4):178-92.

[4] Doyle C, Lennox L, Bell D. A systematic review of evidence on the links between patient experience and clinical safety and effectiveness. BMJ Open. 2013 Jan 3;3(1):e001570.

[5] Kathol DK. Anxiety in surgical patients' families. AORN J. 1984 Jul;40(1):131-7. doi: 10.1016/s00012092(07)69436-8. PMID: 6566535.

[6] Ascher, D.L. (2015), How is she? Information and the surgical waiting lounge. Proc. Assoc. Info. Sci. Tech., 52: 1-8. https://doi.org/10.1002/pra2.2015.145052010047.

[7] Dendere R, et al. Patient Portals Facilitating Engagement With Inpatient Electronic Medical Records: A Systematic Review. J Med Internet Res. 2019 Apr 11;21(4):e12779.

[8] F. Plazzotta, D. Luna, and F. González Bernaldo de Quirós, [Health information systems: integrating clinical data in different scenarios and users], Rev. Peru. Med. Exp. Salud Publica. 32 (2015) 343-351.

[9] M.I. Smith, et al. Lessons Learned After Redesigning a Personal Health Record, Stud. Health Technol. Inform. 245 (2017) 216-220.

[10] Ellis C, Strauss A, Corbin J. Basics of Qualitative Research: Grounded Theory Procedures and Techniques. Contemporary Sociology. (1992)

[11] de Ridder, Else F. et al(2018) "The perioperative patient experience of hand and wrist surgical patients: An exploratory study using patient journey mapping," Patient Experience Journal: Vol. 5 : Iss. 3

[12] Doyle C, Lennox L, Bell D. A systematic review of evidence on the links between patient experience and clinical safety and effectiveness. BMJ Open 2013;3:e001570. doi:10.1136/bmjopen-2012-001570

[13] A Pearson, M Richardson, M Cairns - Ambulatory Surgery, 2004. Best practice" in day surgery units: a review of the evidence. Ambulatory Surgery: Volume 11, Issues 1-2, 2004

[14] Rhodes L, Miles G, Pearson A. Patient subjective experience and satisfaction during the perioperative period in the day surgery setting: a systematic review. Int J Nurs Pract. 2006 Aug;12(4):178-92.

[15] Poudel, R., et al. The Effect of Intra-operative Text Messages in Reducing Anxiety Levels Among Family Members of Patients Undergoing Major Musculoskeletal Tumour Surgery. JOIO 54, 2020

[16] Hodge AB, et al. There's an App for That; Improving Communication during Pediatric Cardiothoracic Surgery. Pediatr Qual Saf. 2018 Mar 28;3(2):e055. 